\title{
Simultaneous determination by gas chromatography of the major metabolites in urine of toluene, xylenes and styrene
}

\author{
P. B. VAN ROOSMALEN AND I. DRUMMOND
}

From the Laboratory Services Branch, Occupational Health and Safety Division, Alberta Labour, 10523100 Avenue Edmonton, Alberta, Canada T5J OA8

ABSTRACT A gas chromatographic method has been developed to determine the following metabolites in urine simultaneously if necessary: hippuric acid from toluene or styrene; 3- and 4-methyl hippuric acids from xylenes; phenylglyoxylic acid and mandelic acid from styrene. Heptadecanoic acid is added to the urine as an internal standard and after ethyl acetate extraction from acidic solution, the trimethylsilyl (TMS) derivatives of the metabolites are formed and simultaneously analysed by gas chromatography on $3 \% \mathrm{OV}-1$ on $80 / 100$ gas chrom $\mathrm{Q}$ (flame ionisation detector).

Styrene is metabolised in the human body by oxidation to mandelic acid, phenylglyoxylic acid, or hippuric acid. All three of these metabolites appear in the urine and all three have been used as indicators of human exposure to styrene (Ohtsuji and Ikeda, 1970; Ikeda et al., 1974) although it is not clear which substance is best for this purpose. The situation is complicated by the varying amounts of the three materials reported to be excreted by people not exposed to styrene. Hippuric acid is a major organic component of urine arising from dietary sources such as quinic acid in prunes and the ubiquitous sodium benzoate added to processed food as a mould inhibitor. The variable level of this background excretion, normally about $1 \mathrm{~g} / 1$ (Buchet and Lauwerys, 1973) makes hippuric acid excretion useful only as an indicator of heavy exposure to styrene or toluene which is also metabolised to hippuric acid (Pagnotto and Lieberman, 1967). A further complication is the different methods which have been used to measure the three metabolites. These include colorimetric (Umberger and Fiorese, 1963; Ogata et al., 1969; Ohtsuji and Ikeda, 1970; Ikeda et al., 1974), fluorimetric (Ellman et al., 1961) and gas chromatographic methods (Šedivec and Flek, 1970; Buchet and Lauwerys, 1973; Slob, 1973; Engström and Rantanen, 1974; Buchet et al., 1974; Engström et al., 1976).

Received for publication 11 January 1977

Accepted for publication 5 August 1977
Slob (1973) pointed out that the colorimetric procedures are non-specific and subject to interference from naturally occurring material and various metabolites (Umberger and Fiorese, 1963; Sedivec and Flek, 1970). This gives rise to an intrusive and variable background which complicates interpretation of the results. The gas chromatographic procedures described in the literature are much more specific, and excretion levels of up to $10 \mathrm{mg} / 1$ of mandelic acid and phenylglyoxylic acid (Buchet et al., 1974) are reported for people not exposed to styrene. However, some are timeconsuming with preliminary paper chromatographic work (Slob, 1973), and some use the reagent diazomethane (Sedivec and Flek, 1970; Buchet and Lauwerys, 1973; Buchet et al., 1974) which is explosive, poisonous and carcinogenic (Tomatis, 1976). A great variety of different column materials and chromatographic conditions are described for each metabolite. Accordingly, we sought to simplify the situation by developing a rapid gas chromatographic method for simultaneous determination of all three metabolites of styrene. The method would also be useful for estimation of exposure to toluene and meta- and para-xylene by measuring hippuric acid and the 3- and 4-methylhippuric acids derived from these solvents.

Materials and methods

Urine samples, preserved by addition of $1 \mathrm{~g}$ citric 
acid, were collected in polyethylene bottles at the end of the work shift and stored as quickly as possible in a refrigerator at $+5^{\circ} \mathrm{C}$. The samples were stable for one week under these conditions, but could be kept for longer if frozen. One gram of ammonium sulphate, $0.2 \mathrm{ml} 5 \%(\mathrm{v} / \mathrm{v})$ sulphuric acid, $1.0 \mathrm{ml}$ of urine and $0.10 \mathrm{ml}$ of an internal standard solution of heptadecanoic acid $(10 \mathrm{mg} / \mathrm{ml}$ in absolute ethanol) were placed in a screw-capped tube, mixed, $4.0 \mathrm{ml}$ ethyl acetate added, and the capped tube was then shaken vigorously for two minutes. The layers were separated by centrifugation and $2.0 \mathrm{ml}$ of the organic layer was transferred to a vial, before evaporation to dryness using dry nitrogen, and subsequent addition of $0.10 \mathrm{ml}$ TRI-SIL concentrate (a mixture of hexamethyldisilazane and trimethylchlorosilane, obtained from Pierce Chemical Co., Rockford, Illinois). A teflon-lined cap was placed on the vial, which was placed in a heating block at $75^{\circ} \mathrm{C}$ for five minutes. At the same time, a standard solution was prepared by pipetting $0.5 \mathrm{ml}$ of a solution of $1 \mathrm{mg} / \mathrm{ml}$ each of hippuric acid (BDH Ltd.), mandelic acid (Eastman Kodak), phenylglyoxylic acid (benzoyl formic acid; Aldrich Chemical Co.), 3- or 4-methylhippuric acid (Terochem Laboratories, Edmonton, Alberta), and heptadecanoic acid (Eastman Kodak) in absolute ethanol into a vial. This was then evaporated to dryness and TRI-SIL added in the manner described above. The ethanol solutions of the standards (conveniently premeasured into the vials) were stored in a freezer at $-10^{\circ} \mathrm{C}$ where they are stable for at least six months.
For chromatographic analysis, $1 \mu \mathrm{l}$ of each trimethylsilyl derivative solution was injected into a gas chromatograph (Perkin-Elmer 900, flame ionisation detector). The glass column was $1.2 \mathrm{~m}$ long, with an internal diameter of $2 \mathrm{~mm}$, and was packed with $3 \%$ OV-1 on $80 / 100$ gas-chrom $Q$. The oven temperature was $130^{\circ} \mathrm{C}$ for six minutes, and was adjusted to $215^{\circ} \mathrm{C}$ at a rate of $12^{\circ} \mathrm{C} / \mathrm{min}$. The injector and manifold temperatures were $250^{\circ} \mathrm{C}$; the carrier (nitrogen) gas flow was $20 \mathrm{ml} / \mathrm{min}$, the hydrogen flow $30 \mathrm{ml} / \mathrm{min}$ and the air flow $300 \mathrm{ml} / \mathrm{min}$.

Areas of peaks were measured using a HewlettPackard 3370B integrator and the concentration of the component $\mathrm{X}$ in the urine calculated as follows:

$$
\begin{aligned}
& X(g / l)=\frac{R_{\mathbf{x}} \cdot C_{\mathbf{x}}}{\mathbf{R}_{\mathbf{s}} \cdot \mathbf{C}_{\mathbf{s}}} \times \frac{\text { Total volume of urine taken }}{\text { Volume of urine used }} \\
& \text { where } \\
& \mathbf{R}_{\mathbf{x}}=\frac{\text { area } X \text { in sample }}{\text { area internal standard in sample }} \\
& \mathbf{R}_{\mathbf{s}}=\frac{\text { area } X \text { in standard }}{\text { area internal standard in standard }} \\
& \mathbf{C}_{\mathbf{B}}=\frac{\text { concentration } X \text { in standard }}{\text { concentration internal standard in stand. }} \\
& \mathbf{C}_{\mathbf{x}}=\text { concentration internal standard in sample }
\end{aligned}
$$

This result was then corrected to a mean specific gravity of 1.024 using the formula,

$$
X(\text { corrected })=X \times \frac{0.024}{\text { specific gravity }-1} \mathrm{~g} / 1
$$

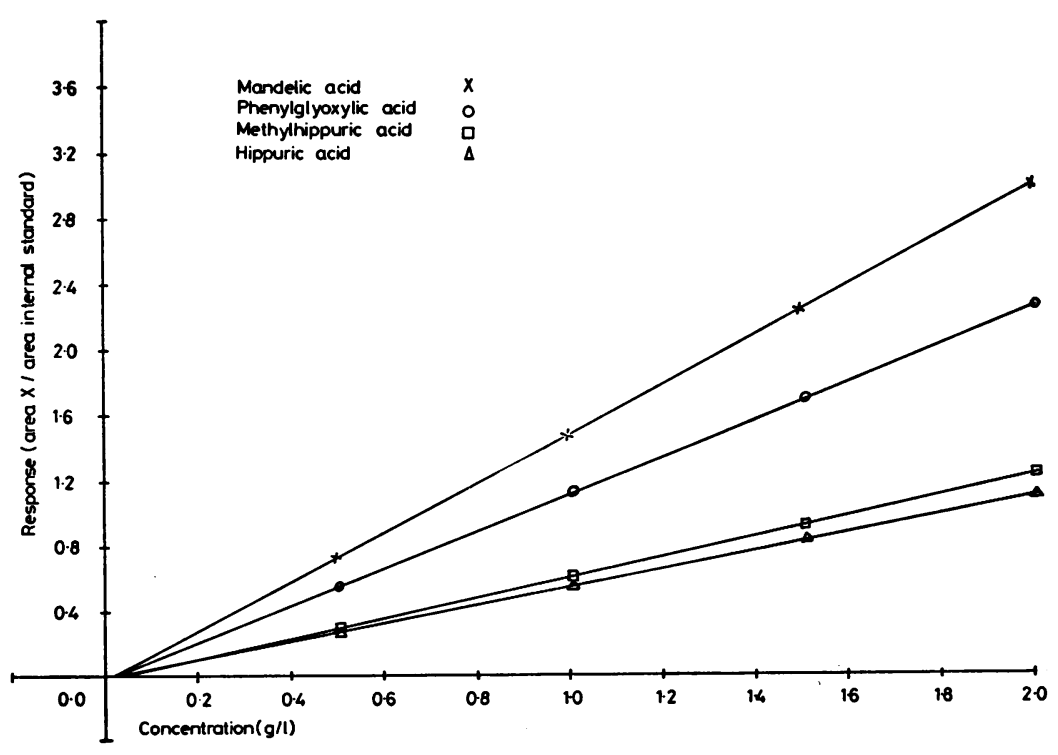

Fig. 1 Responses of the four metabolites from urine samples for various levels of addition of each component. The background level of $0 \cdot 8 \mathrm{~g} / \mathrm{l}$ has been subtracted from the measured values for hippuric acid. 
to compensate for the different urine volumes from different individuals (Elkins and Pagnotto, 1965). The specific gravity of the urine was measured by refractometer and corrected for the increase caused by adding approximately $1 \%(w / v)$ of citric acid as a preservative.

\section{Results and discussion}

Each step of our analytical procedure was tested. We found in the extraction phase that it was

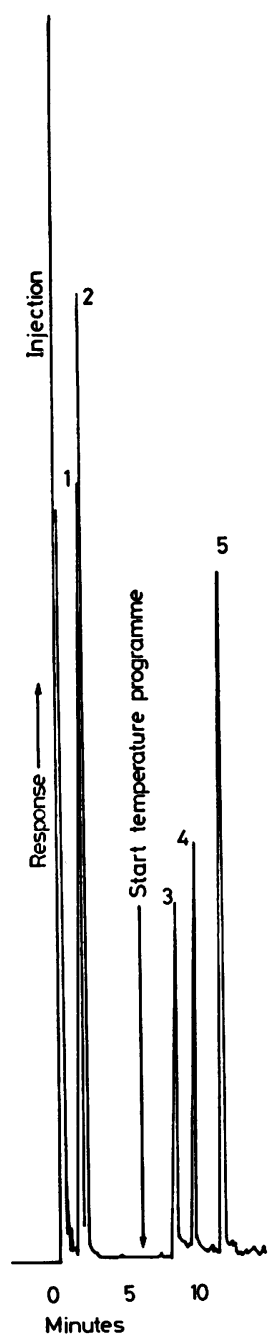

Fig. 2 Chromatogram of the standard mixture. The concentration of each component is $1 \mathrm{mg} / \mathrm{ml} .1$ = phenylglyoxylic acid; 2 = mandelic acid; 3 = hippuric acid; 4 = 3-methylhippuric acid; $5=$ heptadecanoic acid (internal standard). important to make the urine strongly acid, not to hydrolyse conjugated forms of the metabolites as has been suggested (Slob, 1973) but merely because the salts of the acids do not extract into ethyl acetate. The efficiency of the extraction procedure was tested by analysing the extract by gas chromatography (external standard method), when it was found that the presence of ammonium sulphate and volume of ethyl acetate were important. Under the conditions described above we performed 16 ex-

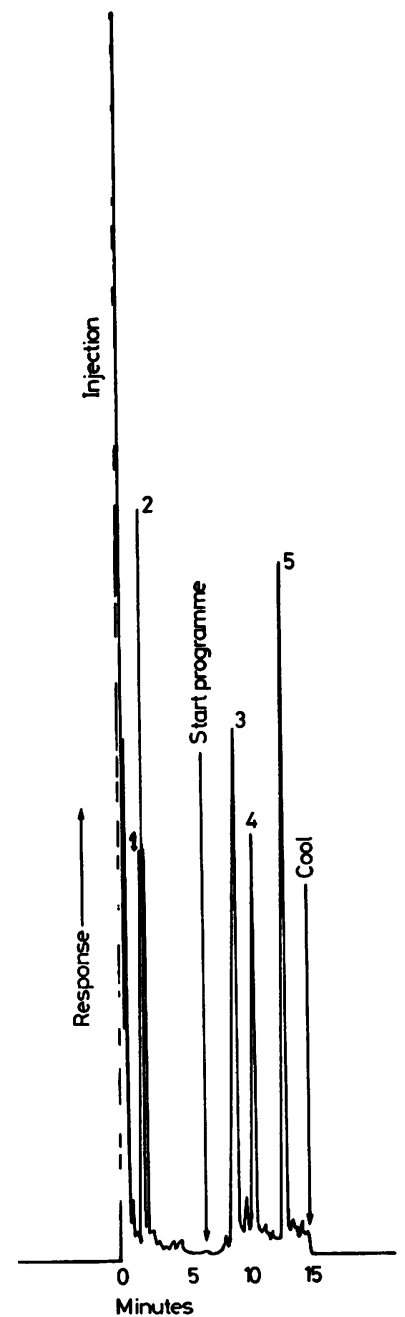

Fig. 3 Chromatogram of a urine sample obtained from a worker exposed to styrene. The following components were measured: 1 , phenylglyoxylic acid, $0.36 \mathrm{~g} / \mathrm{l} ; 2$, mandelic acid, $0.95 \mathrm{~g} / \mathrm{l} ; 3$, hippuric acid, $1.2 \mathrm{~g} / \mathrm{l} ; 4$, 3-methylhippuric acid added at a level of $1.0 \mathrm{~g} / \mathrm{l} ; 5$, heptadecanoic acid, internal standard. 
tractions on all five components of the standard mixture and found mean recoveries of $97-99 \%$ with coefficients of variation ranging from $1 \cdot 5-5 \%$.

When making the derivatives, the time of heating was varied from five to 30 minutes. No difference was found between the treatments and so the shortest time was chosen. The overall analytical procedure was tested by performing recoveries of the metabolites from spiked urines. The results are shown in the form of calibration graphs in Fig. 1.

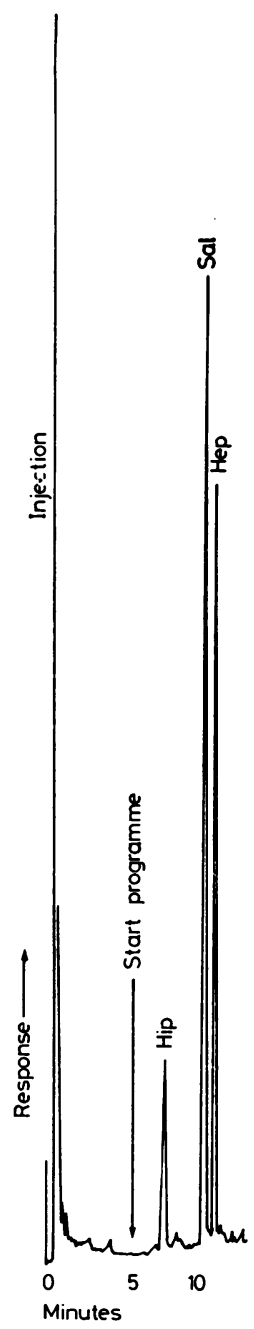

Fig. 4 Chromatogram of a urine sample from a person not exposed to styrene but who had taken $750 \mathrm{mg}$ acetylsalicylic acid the previous evening. Hip = hippuric acid, $0.5 \mathrm{~g} / \mathrm{l} ; \mathrm{Sal}=$ salicyluric acid (aspirin metabolite) $; \mathrm{Hep}=$ heptadecanoic acid, internal standard.
Chromatograms of the standard mixture and of urine of a worker exposed to styrene are shown in Figs. 2 and 3. We estimate the limits of detection of the metabolites in urine to be $4 \mathrm{mg} / 1$ for methylhippuric acid; $2 \mathrm{mg} / \mathrm{l}$ for mandelic acid, and $2 \mathrm{mg} / \mathrm{l}$ for phenylglyoxylic acid. A detection limit is not given for hippuric acid for, as noted above, it is a major constituent of urine. In the urine of people not exposed to toluene, xylene or styrene the following background concentration of the metabolites, corrected to a specific gravity of 1.024 have been found in this laboratory: hippuric acid, 0.5-1.2 g/1; mandelic acid up to $5 \mathrm{mg} / \mathrm{l}$; phenylglyoxylic acid and the methylhippuric acids have not been detected.

As acetylsalicylic acid is a widely used drug we investigated whether its metabolites interfered with this determination. Fig. 4 shows a chromatogram from the urine of a person not exposed to solvents, who had taken two tablets, each containing $375 \mathrm{mg}$ of acetylsalicylic acid, the previous evening. The major peak attributable to the drug metabolite, salicyluric acid, does not interfere with the determination of the styrene, toluene or xylene metabolites.

In summary, we believe that this method of determining these metabolites offers significant advantages over those methods described in the literature. It is more selective than colorimetric and u.v. methods, more accurate by virtue of using an internal standard and uses less toxic chemicals. A significant saving in time is accomplished by using one extraction, one type of derivative and one column for all four metabolites.

\section{References}

Buchet, J. P., and Lauwerys, R. R. (1973). Measurement of urinary hippuric and m-methylhippuric acids by gas chromatography. British Journal of Industrial Medicine, 30, 125-128.

Buchet, J. P., Lauwerys, R. R., and Roels, H. (1974). Évaluation de l'exposition des travailleurs au styrène par le dosage de ses métabolites urinaires: les acides mandélique et phénylglyoxylique. Archives des Maladies Professionnelles de Médecine du Travail et de Sécurité Sociale (Paris), 35, 511-516.

Elkins, H. B., and Pagnotto, L. D. (1965). Is the 24-hour urine sample a fallacy? American Industrial Hygiene Association Journal, 26, 456-460.

Ellman, G. L., Burkhalter, A., and Ladou, J. (1961). A fluorometric method for the determination of hippuric acid. Journal of Laboratory and Clinical Medicine, 57, 813-818.

Engström, K., Husman, K., and Rantanen, J. (1976). Measurement of toluene and xylene metabolites by gas chromatography. International Archives of Occupational and Environmental Health, 36, 153-160.

Engström, K., and Rantanen, J. (1974). A new gas chromatographic method for determination of mandelic acid in urine. Internationales Archiv für Arbeitsmedizin, 33, 163-167. 
Ikeda, M., Imamura, T., Hayashi, M., Tabuchi, T., and Hara, I. (1974). Evaluation of hippuric, phenylglyoxylic and mandelic acids in urine as indices of styrene exposure. Internationales Archiv für Arbeitsmedizin, 32, 93-101.

Ogata, M., Tomokuni, K., and Takatsuka, Y. (1969). Quantitative determination in urine of hippuric acid and $\mathrm{m}$ - or p-methylhippuric acid, metabolites of toluene and $\mathrm{m}$ - or p-xylene. British Journal of Industrial Medicine, 26, 330-334.

Ohtsuji, H., and Ikeda, M. (1970). A rapid colorimetric method for the determination of phenylglyoxylic and mandelic acids, and its application to the analysis of urine of workers exposed to styrene vapour. British Journal of Industrial Medicine, 27, 150-154.

Pagnotto, L., and Lieberman, L. M. (1967). Urinary hippuric acid excretion as an index of toluene exposure. American Industrial Hygiene Association Journal, 28, 129-134.

Sedivec, V., and Flek, J. (1970). Bestimmung toxischer Substanzen und ihrer Metaboliten in Biologischen Flüssig keiten mittels der Gas chromatographie. V. Hippursäure im Urin. Collection of Czechoslovak Chemical Communications, 35, 3265-3273.

Slob, A. (1973). A new method for determination of mandelic acid extraction at low level styrene exposure. British Journal of Industrial Medicine, 30, 390-393.

Tomatis, L. (1976). The IARC program on the evaluation of the carcinogenic risk of chemicals to man. Annals New York Academy of Sciences, 271, 396-409.

Umberger, C. J., and Fiorese, F. F. (1963). Colorimetric method for hippuric acid. Clinical Chemistry, 9, 91-96. 